\title{
Inteligencia emocional y liderazgo
}

\section{Emotional intelligence and leadership}

Ing. Jorge Brito, MBA.

Universidad Internacional del Ecuador, Ecuador

Autor para correspondencia: jurgenbritto@gmail.com

Fecha de recepción: 17 de marzo de 2016 - Fecha de aceptación: 14 de abril de 2016

\section{Resumen}

Revisando la literatura sobre liderazgo y su relación con elementos tales como personalidad e inteligencia emocional de manera particular. Este estudio propone preguntas y también la necesidad en profundizar sobre este tema que tiene una implicación directa en el relacionamiento con las personas del mismo país, de la región y del mundo en general con miras al desarrollo de nuestras empresas a nivel global.

Palabras claves: inteligencia emocional; liderazgo; negocios

\begin{abstract}
Reviewing the literature on leadership and its relationship with elements such as personality and emotional intelligence in a particular way. This study proposes questions and the need to deepen on this issue that has a direct involvement in the relationship with people of the same country, the region and the world in general for the development of our businesses globally.
\end{abstract}

Key words: emotional intelligence; leadership; business 


\section{Introducción}

Durante el transcurso de nuestra vida profesional, con seguridad, todos hemos sido testigos de algún asenso de un colega de trabajo o hemos sido ascendidos a cargos de mayor responsabilidad o cómo líderes de equipos de trabajo, también hemos participado en reuniones profesionales y personales en dónde quizás hemos escuchado "por qué ascienden a tal o cual persona...". Estas reflexiones ponen en la palestra algunas preguntas que se plantean profesionales y ejecutivos: ¿Qué atributos hay que tener para que me tomen en cuenta para un ascenso?, ¿Por qué no me eligen a mí que tengo más experiencia?, "He dejado parte de mi vida en esta empresa, ¿Cuándo seré promovido a cargos de responsabilidad? Este tipo de preguntas surgen frecuentemente en ejecutivos de empresas públicas y privadas y en éstas últimos ejecutivos que colaboran en empresas transnacionales y familiares. Paralelamente, preocupación similar debe existir en accionistas, gerentes generales, dueños y otros funcionarios responsables de elegir a un líder para que lleve a las empresas a buen puerto y genere el valor esperado por los grupos de interés.

Una mirada retrospectiva al pasado, nos plantea la necesidad de entender qué aspectos del profesional son más relevantes o qué rasgos priman al momento de elegir un líder empresarial. La creencia popular de que los resultados son los únicos elementos que se consideran para la delegación no se cumplen necesariamente en todas las organizaciones y particularmente en las empresas familiares del Ecuador.

Revisando la literatura sobre liderazgo y su relación con elementos tales como personalidad e inteligencia emocional de manera particular, me surgen muchas preguntas y también la necesidad en profundizar sobre este tema que tiene una implicación directa en el relacionamiento con las personas del mismo país, de la región y del mundo en general con miras al desarrollo de nuestras empresas a nivel global.

Aunque el término inteligencia emocional (IE) es acuñado en 1990 John Mayer y Peter Salovey quienes definieron IE como "una forma de inteligencia social que involucra la habilidad de detectar las emociones propias y las de los otros para analizarlas y emplearlas como guía de pensamiento y acción", la IE no es nueva comenta el profesor de Sicología de la universidad Internacional del Ecuador en una entrevista con el autor de este ensayo. Las investigaciones sicológicas sobre la inteligencia tradicionalmente se enfocaban a aspectos cognitivos, no obstante, otros autores aseguraban que las habilidades no intelectuales eran esenciales para conocer la capacidad de una persona para resolver problemas cotidianos y comportarse inteligentemente (Wechsler, 1943). Las conclusiones a las que llegaron éste y otro grupo de estudiosos del tema era que los líderes capaces de establecer confianza, respeto y calidez hacia sus subordinados logran mejores resultados.

Estudios diseñados para detectar la IE han demostrado que las personas que tenían mejor desarrollada esta capacidad se recuperan con mayor rapidez de situaciones desagradables y se adaptan con mayor facilidad a los cambios.

¿Inteligencia Emocional o Inteligencia Cognitiva?, estudios varios han llegado a la conclusión de que la inteligencia cognitiva (IQ) no está directamente relacionada con un buen desempeño laboral y que ésta cuenta en el mejor de los casos con un 30\% (Sternberg, 1996). Y no es que e IQ sea 
irrelevante, pero una vez que se consiguió empleo -gracias a los conocimientos adquiridos- el factor predominante para tener éxito está más relacionado con aspectos sociales y emocionales (Goleman, 1998).

En las empresas ecuatorianas, transnacionales y familiares en especial, toman muy poco en cuenta el equilibrio emocional de los empleados, "cuesta mucho convencer a la gente la importancia de las emociones en el trabajo, pues los directivos sólo están interesados en apoyar la formación de sus ejecutivos con foco a la productividad, procesos, aspectos contables, normas, marketing, ventas, finanzas y otro tipo de formación que "genere resultados en corto plazo" comenta Mauricio Valdivieso, Director Corporativo de Talento Humano del Grupo Nobis de Guayaquil-Ecuador durante una entrevista para el desarrollo de este ensayo.

El manejo de las emociones es uno de los aspectos fundamentales del líder actual. Las empresas públicas y privadas de los países en desarrollo, requieren líderes con habilidades de relacionamiento extraordinarias, con capacidad de adaptación social y cultural, capaces de agradar y contagiar a sus subordinados, auténticos para proponer metas desafiantes y motivar mas con su accionar que con sus discursos.

La inteligencia emocional (IE) es una de las aportaciones más importantes al mundo de los negocios (D'Alessio, 2010). Actualmente, existe mucha literatura al respecto para definir la IE, la revisada por el autor de este ensayo, topa o se refiere un conjunto de habilidades humanas para manejar sus emociones, aceptar y entender las de otros y a través de su discernimiento actuar o tomar decisiones en su entorno.

Los objetivos de este ensayo se orientan al conocimiento y evaluación de la inteligencia emocional con el liderazgo y de qué manera mejora el desempeño de las personas (profesionales, ejecutivos, empresarios, entre otros) para trabajar en equipo. Autores como Palmer, Walls, Burgess y Stough (2001) consideran a la inteligencia emocional como una medida efectiva y de gran reconocimiento en el mundo académico y empresarial para identificar a los líderes efectivos. Sin duda alguna, el conocimiento de éstos elementos o factores que influyen o inciden en la eficiencia de líderes, ejecutivos y empresarios en general permitirá la adaptación o mejoramiento de las mallas curriculares de los centros de estudio y en general centro de capacitación.

El presente ensayo, presenta varios estudios referentes a la relación entre IE y liderazgo. Los aspectos humanos, tales como personalidad, inteligencia, emociones, entre otros, han sido estudiados por la sicología. Se considera que en el periodo 1990-1993 surge la IE y fue definida por Mayer y Salovey (1993) como "un tipo de inteligencia social que involucra la habilidad de monitorear las emociones propias y las de otros, distinguirlas entre ellas, y usar la información para guiar el pensamiento y las acciones de uno" (p.433). Esta definición de lógica aparente trae consigo importantes desafíos para los investigadores, quienes han dedicado muchas horas para descubrir la forma más apropiada para conocer y medir la IE en cada persona, teniendo presente un principio generalmente aceptado como es la individualidad y libertad del ser humano. A partir del año 1993, estos autores han ido reformulando el concepto inicial con nuevas aportaciones (Mayer y Salovey, 1993, 1997, 2007; Mayer, Caruso y Salovey, 1999, 2001; Mayer, Salovey y Caruso, 2000).

Uno de los primeros modelos el propuesto por Mayer y Salovey (1993) se denomina modelo de las cuatro ramas de la inteligencia emocional, donde estas ramas están 
interrelacionadas. La rama 1, percibir emociones [Perceiving Emotions (PEIQ)], es la habilidad para percibir, identificar, valorar y expresar emociones propias y las de los demás, a través del lenguaje y conducta, así como la percepción de emociones en objetos, arte, historias, música y otros estímulos; la rama 2, facilitación emocional del pensamiento [Facilitating Thought (FEIQ)], es la habilidad de genera, usar, y sentir las emociones como necesarias a fin de comunicar los sentimientos o emplear las emociones en otros procesos cognitivos. El estado de humor cambia la perspectiva del individuo, desde el optimismo al pesimismo, favoreciendo la consideración de múltiples puntos de vista.

Los estados emocionales facilitan el afrontamiento. Por ejemplo, el bienestar facilita la creatividad; la rama 3, comprender las emociones [Understanding Emotions (UEIQ)], es la capacidad de comprender y analizar las emociones, las señales emocionales en las relaciones interpersonales son comprendidas y tienen implicaciones en la misma relación, por ejemplo: la tristeza se podría deber a una pérdida, la UE también es la habilidad para comprender sentimientos complejos, por ejemplo: el amor y odio simultáneo a una persona querida (pareja, hijos) durante un conflicto; la rama 4, manejo de las emociones [Managing Emotions (MEIQ)] es la habilidad para estar abierto a los sentimientos y modularlos en uno mismo y en los demás, capacidad para mitigar las emociones negativas y potenciar las positivas, sin reprimir o exagerar la información que transmiten.

La escala de las cuatro ramas evolucionó, Mayer, Salovey y Caruso (2002) presentaron al test MSCEIT con el mismo propósito. Este test se conforma de dos áreas: 1) inteligencia emocional experiencial (EEIQ), y 2) inteligencia emocional estratégica (SEIQ).

Goleman (1995), es uno de los investigadores que mayor aporte han realizado al estudio de la efectividad del liderazgo, en 1998 declara que "el coeficiente intelectual (CI) y las habilidades técnicas son importantes, pero que la IE es el sine qua non del liderazgo". Goleman (1998) propuso los cinco componentes de la IE: 1) Autoconciencia (consciencia de sí mismo) es la capacidad de reconocer y entender sus emociones, estado de ánimo e impulsos, así como su efecto en los demás; los rasgos más representativos en las personas con este distintivo son: la confianza en sí mismo, autoevaluación realista, sentido del humor autocrítico; 2) Autodominio (autorregulación) es la habilidad para controlar o redirigir impulsos y estados de ánimo, propenso a evitar juicios de valor y en general, pensar antes de actuar, los rasgos distintivos son: integridad, confianza y abierto al cambio; 3) Motivación que es la capacidad para lograr metas y trabajar por motivos de mayor categoría que el dinero, los distingue el optimismo, su compromiso con las organizaciones y foco a resultados; 4) Empatía es la habilidad para tratar a las personas de acuerdo a sus reacciones emocionales, el servicio a los demás, la sensibilidad cultural y su capacidad para interrelacionarse con los demás para fomentar el trabajo son sus principales distintivos; 5) Habilidad Social, es la habilidad para fomentar las relaciones con los demás de manera agradable y simpática, son personas expertas en el manejo de equipos, persuadir y fomentar cambios organizacionales.

Las dimensiones de la inteligencia emocional y sus competencias han venido evolucionando y han sido racionalizadas. A continuación, nos referiremos a los estudios más representativos presentados por D’Alessio en su libro. En él se recogen los estudios más recientes sobre IE que nos servirán de base para el análisis de los paperes relacionados. 
Goleman, Boyatzis y McKee (2002) presentan los siguientes dominios de la IE y sus competencias: la competencia personal compuesta por la consciencia en sí mismo (autoconsciencia) y la autogestión, y la competencia social compuesta por la consciencia social y las habilidades sociales. Mayer, Salovey, Caruso y Sitarenios (2001, 2003) presentaron argumentos razonables para medir la IE con su análisis factorial confirmatorio (MSCEIT) que apoyó el modelo teórico de la IE. Dulewicz y Higgs (2003) estudiaron la necesidad de la IE en las organizaciones, éstos autores utilizaron siete elementos principales de la IE: autoconsciencia, capacidad de recuperación emocional, motivación, sensibilidad interpersonal, influencia, intuición, y escrupulosidad. Otras pruebas usadas para medir la relación entre el liderazgo y la IE es la Prueba de IE de la universidad de Swinburne [Swinburne University Emotional Intelligence Test (SUEIT)] (Palmer y Stough, 2001). A través de los estudios realizados con este test, autores como Gardner y Stough (2002) concluyeron que la inteligencia emociona se correlacionó altamente con todos los componentes del liderazgo transformacional, por lo tanto, se reconoce a los líderes efectivos como aquellos que reportan comportamientos transformacionales (Gardner y Stough, 2002).

Estudios posteriores, realizados a un grupo de estudiantes universitarios, a los cuales se les aplicó un cuestionario que contenía: 1) una medida de inteligencia emocional, 2) una medida de inteligencia social y 3) una medida de liderazgo. Las conclusiones de estos estudios sugieren la importancia de estos dos componentes: sociales y emocionales (Kobe, Reiter-Palmon y Rickers, 2001). Otra de las preocupaciones de los investigadores, han sido la relación entre la IE y el desempeño de los líderes en los equipos de trabajo, para el efecto Prati, Douglas, Ferris, Ammeter y Buckley, 2003) presentaron el modelo de inteligencia emocional y efectividad del líder del equipo, el mismo que ha sido cuestionado por varios autores. Sin embargo, los pocos estudios al respecto, como el realizado por Barling, Slater y Kellowat (2000) muestran que la influencia idealizada, la motivación inspiracional, y la consideración individualizada diferían de acuerdo a los niveles de inteligencia emocional de los participantes.

La inteligencia emocional es una gran contribución para el manejo gerencia, su relación con otros elementos estudiados en el ser humano, tales como el pensamiento crítico y los estilos de liderazgo (D’Alessio, 2010). Los estudios narrados en este ensayo, sugieren continuar con investigaciones al respecto por la importancia que podría representar este tipo de investigaciones para estimar los estilos de liderazgo a partir del conocimiento de la IE de los profesionales y ejecutivos en las organizaciones. Para los países en desarrollo, como es el caso de Ecuador, constituye una enorme oportunidad para replicar o verificar si los modelos diseñados en países desarrollados se ajustan a las realidades de nuestros países, teniendo en cuenta que más del $83 \%$ de las empresas ecuatorianas, colombianas y peruanas con de tipo familiar.

\section{Bibliografía}

Barling, J., Slater, F., \& Kellowat, E. K. (2000). Transformational leadership and emotional intelligence: An exploratory study. Leadership \& Organization Development Joumal, 21(3), $157-161$.

Cherniss, C. (2005). Inteligencia Emocional en el Trabajo. 3ra. Ed. Barcelona: Editorial Kairos. 
D’Alessio, F. (2010), Liderazgo y Atributos Gerenciales: Una visión Global y Estratégica, México, Pearson Educación Prentice Hall

Dulewicz, V., \& Higgs, M. (2003). Leadership at the top: The need for emotional Intelligence in organizations. The International Journal of Organizational Analysis, 11(3), 193-210.

Goleman, D. (1995). Emotional intelligence: Why It can matter more than IQ. New York: Batman.

Goleman, D. (1998). "La práctica de la Inteligencia Emocional: La tan esperada continuación del BestSeller Inteligencia Emociona. Barcelona: Editorial Kairos,

Goleman, D., Boyatzis, R., \& McKee, A. (2002). Primal leadership: Realizing the power of emotional intelligence., Harvard Business School. Boston. Press.

Kobe, L. M., Reiter-Palmon, R., \& Rickers, J.D. (2001). Self-reported leadership experiences in relation to inventoried social and emotional Intelligence. Current Psychology, DevelopmentalLearning-Personality-Social, 20 (2), 154-163.

Mayer, J. D., \& Salovey, P. (1993). The intelligence of emotional intelligence. Intelligence, 1(17), 442443.

Mayer, J. D., Salovey, P., \& Caruso, D. R. (2002). Mayer-Salovey-Caruso emotional Intelligence test (MSCEIT). User's manual North Tonawanda, NY: Multi-Health Systems

Mayer, J. D., Salovey, P., Caruso, D. R., \& Sitarenios, G. (2001). Emotional Intelligence as a Standard Intelligence. Emotion, 1(3), 232-242.

Mayer, J. D., Salovey, P., Caruso, D. R, \& Sitarenios, G. (2003). Measuring Emotional intelligence with the MSCEIT v. 2.0. Emotion, 3(1), 97-105

Gardner, L., \& Stough, C. (2002). Examining the relationship between leadership and emotional intelligence in senior level managers. Leadership \& Organizational Development Journal, 23(1/2), 68-78.

Palmer, B., \& Stough, C. (2001). Workplace SUEIT: Swinburne University Emotional Intelligence Test-Descriptive Report. Organization Psychology Research Unit. Australia: Swinburne University.

Palmer, B., Walls, M., Burgess, Z., \& Stough, C. (2001). Emotional intelligence. Leadership \& Organizational Development Joumal, 22(1), 5-10.

Prati, L. M., Douglas, C., Ferris, G. R., Ammeter, A. P., \& Buckley, M. R. (2003a). Emotional intelligence, leadership effectiveness, and team outcomes. The International Joumal of Organizational Analysis, 11(1), 21-40.

Sternberg, R. J. (1996), Intelligence: Knows and Unknows, American Psychologist, 51(2), 77-101.

Wechsler, D. (2003), Wechsler Intelligence Scale for Childern. Pearson, Cuarta Edición. 